\title{
Physical education interventions improve the fundamental movement skills in kindergarten: a systematic review and meta-analysis
}

\author{
Bin $\mathrm{LI}^{1,2}$, Jing $\mathrm{LIU}^{* 1}\left(\mathbb{D}\right.$, Binbin YING ${ }^{3}$
}

\begin{abstract}
Fundamental motor skills (FMS) are the foundation for the development of advanced motor skills. To assess the effect of physical activity intervention on the promotion of FMS and explore the influencing factor of physical activity intervention on the development of FMS in preschool children. Trails with children aged 3-7yeas in kindergarten settings that applied physical activity intervention programs were included. We calculated the effects on overall FMS, locomotor skill and object control skill subscales (LMS and OCS) by weighted standardized mean differences using random-effects models. In this study, 23 trials involving 2258 preschoolers (aged 3.3-6.5years) were selected, including 7 high quality studies, 11 medium quality studies and 5 low quality studies. Analysis of the effects of physical activity intervention revealed significant differences among groups in favor of intervention group, FMS (SMD = 1.50, 95\% CL [1.29, 1.71], $I^{2}=89.7 \%$ ), locomotor skills ( $\mathrm{SMD}=1.56,95 \% \mathrm{CL}$ $\left.[1.38,1.75], I^{2}=59.2 \%\right)$, object control skills ( $\mathrm{SMD}=1.43,95 \% \mathrm{CL}[1.25,1.61], I^{2}=93.8 \%$ ). Physical activity interventions can significantly improve FMS in healthy preschool aged children. However, we should be cautious due to the limited researches, low-quality evidence and immediate post-intervention effects without long-term follow-up.
\end{abstract}

Keywords: fundamental motor skill; preschool children; physical activity; meta-analysis.

Practical Application: Fundamental motor skills (FMS) are the foundation for the development of advanced motor skills. In this study, we assessed the effect of physical activity intervention on the promotion of FMS and explored the influencing factor of physical activity intervention on the development of FMS in preschool children. Our results showed that physical activity interventions can significantly improve FMS in healthy preschool aged children.

\section{Introduction}

Fundamental motor skills are classified into locomotor skills that enable children to move the body in space and object control skills that enable to manipulate and project objects (Cools et al., 2009). FMS are the foundation movements such as running, catching and jumping, which form the building blocks for more complex and specialized skills which are essential for participation in physical activities and sports and recreation. At present, developmental coordination disorder (DCD), attention deficit hyperactivity disorder (ADHD), autism, and obesity in preschool children have become more and more prominent (Bryant et al., 2014; Meng et al., 2018; Wen, 2017; Kang et al., 2017). However, there are significant correlations with fundamental motor skills (Robinson et al., 2015). More recent evidence has shown the association of higher levels of FMS with better overall health outcomes (D'Hondt et al., 2013), including a lower body mass index and higher cardiorespiratory fitness (Fong et al., 2012), obesity prevention (Wang et al., 2018) and cognitive function improvement (Lloyd et al., 2014). fundamental motor skills are positively correlated with physical activity levels (Tortella et al., 2016), and there is a strong positive correlation between the proficiency of fundamental motor skills at the age of 6 and the level of physical activity at the age of 26 (Veldman et al., 2017).

In the past, several studies on physical activity intervention on improving FMS have been published. Vassiliki et al. presented that participation in physical activity of teacher-leading interventions for about 10 weeks can significantly promote FMS development in preschool children compared with free activities (Iivonen et al., 2011; Burrows et al., 2014; Bonvin et al., 2013). However, Burrows et al. found that the effect of physical activity intervention on improving preschool children's FMS is not significantly different (Jones et al., 2016; Moher et al., 2015; Higgins \& Green, 2008). Although two reviews both found that intervention were effective in improving FMS, these articles were methodologically failed to provide solid evidence of the effectiveness of FMS intervention in preschool children. One of these systematic reviews included 20 studies showing that physical activity of teacher-leading intervention produced effect sizes in the range of $0.14-0.47$ for overall FMS, OCS, or LMS. The greatest limitation of the included articles was the lack of blinding without consistencies in the frequency and duration of activity sessions. The other review showed that $60 \%$ of the 
included studies revealed statistically significant intervention effects, but the meta-analysis was not conducted for the low methodological quality and large heterogeneity. Therefore, the exact effect of physical education intervention on preschool children's FMS is unclear. Our study aims performed systematic review and meta-analysis to describe and evaluate physical education program intervention. Meanwhile, meta-regression to analyze the influence factor of physical education improve FMS for health preschool children was conducted. We further performed subgroup analysis to confirm the influencing factor. Finally, we performed exploratory analyses to identity the intervention that were more effective than others.

\section{Method}

This review and meta-analysis were performed in accordance with the guidelines outlined in the preferred reporting item for systematic reviews and meta-analyses (PRISMA) statement (Zhu, 2015).

\subsection{Literature search}

An electronic database search was conducted using Medline, Embase, Pubmed, Eric, Cochrane library, Web of Science, China national knowledge infrastructure, digital journal of Wanfang Data, China Science and Technology Journal Database from the establishment of the database to May 31, 2020. Based on the PICOS approach, search terms included: population (preschool* OR child* OR kindergarten OR "early child ${ }^{\star}$ " $\mathrm{O}$ $\mathrm{R}$ "young child*"), intervention: ("structured physical activity" OR "physical activity" OR "physical education" OR exercise), Comparator (Non-structured sports activities), outcome ("motor skill" OR "fundamental motor skill" OR "fundamental movement skill” OR “object control skill” OR "locomotor skills" OR "gross motor skill", Study design ("controlled trial" OR "randomized controlled trial” OR "Cluster randomized controlled trial”).

\subsection{Eligibility criteria}

Inclusion criteria: (1) Peer-reviewed controlled experimental studies published in English and Chinese before May 31, 2020. (2) Samples were composed of healthy preschool children (age 3-7 years). (3) Experimental group participates in planned, purposeful, and organized structured sports activities by teacher lead, while the control group students engage in non-structural free activities. (4) Quantitative assessment of the results of fundamental motor skills. (5) The trail had to report a fundamental motor skill outcome measure in both groups. The outcome indicators include at least one of the total score of motor skills development, mobile skills, and the object control skills.

Exclusion criteria: (1) Excluding research with subjects related to without major health or motor handicaps. such as developmental delay and attention deficit hyperactivity disorder. (2) Excluding studies with less than 4 weeks of intervention and less than 10 subjects. (3) Exclude non-English or Chinese related research. Review papers, uncontrolled trials.

\subsection{Study selection and data extraction}

Two authors (Li and Ying) worked independently and checked in pairs the eligibility status of identified citations by screening titles, abstracts, and then the full paper. Two reviewers used a standard form to extract information from each eligible study, including research characteristics (such as author, country, year), participants and cluster demographics (such as sample size, age, gender), intervention details (such as duration, frequency and content of intervention), study methodology (such as randomization, blinding, allocation concealment), outcome date, etc. In case of any disagreement, consensus was reached through discussions and also by including a third person.

\subsection{Quality evaluation}

The reviewers (Li and Ying) assessed the risk of bias of each eligible study using Cochrane bias risk assessment tool (Engel et al., 2018). Specific indicators included: selection bias, implementation bias, measurement bias, dropout or loss to followup, reporting bias and other biases, among which other biases include less than 30 cases and are determined to be high-risk, while 30 cases or more are recognized as low risk. If the sample size is not reported, then the risk of bias is determined to be unclear. Research evaluation is divided into 'low risk', 'unclear' and 'high risk'. The research quality was divided into three levels from high to low, including Level A (low bias): Fully meet 4 items or more, Level B (Moderate bias): satisfy 2-3 items, and Level C (highly biased): only satisfy 1 item (Higgins \& Green, 2008) Two reviewers independently scored the items for each study. If final results of the two investigators are in dispute, we would consult the third investigator (Liu). We will contact the author via email to get the missing data as much as possible if the data information of research is not complete.

\subsection{Statistical analysis}

Meta-analyses were completed for continuous data by using the change in the mean and standard deviation of outcome measures. If not provided, the change in post-intervention mean was calculated by subtracting baseline from post-intervention values. Change in the standard deviation of post-intervention outcomes was calculated using Revman 5.3. Data required was (1) $95 \%$ confidence interval data for pre/post intervention change within groups, or when this was not available, (2) actual $p$ values for pre/post intervention change within groups, or if only the level of significance was available, (3) then we used default $p$-values where $p<0.05$ becomes $p=0.049, p<0.01$ becomes $p=0.0099$ and $p=$ not significant becomes $p=0.05$. A random effects inverse variance was used with the effects measure of SMD (Higgins \& Thompson, 2002). We used Stata13.0 software to conduct a combined analysis of the outcome indicators of the included literature, Chi-square test was used to test the heterogeneity between studies, and $P>0.01$ means no statistical difference or no heterogeneity (Demets, 1987). At the same time, the $I^{2}$ value was used for quantitative evaluation, while $I^{2}<40 \%$ means low heterogeneity, $40 \% \leq I^{2} \leq 70 \%$ is moderately heterogeneous, and $I^{2}>70 \%$ is highly heterogeneous (Stuck et al., 1998). We used a fixed model for low heterogeneity and a random effects model for medium or high heterogeneity (Adamo et al., 2016). Also, we explored the source of heterogeneity through meta regression and subgroup analysis, and applied Egger to analyze publication 
bias (Robinson et al., 2016), and then created the corresponding funnel chart.

\section{Results}

\subsection{Research characteristics}

Overall, we identified 7794 studies and an additional 18 studies were identified through other sources to give a total of 7872 studies, in which we assessed 78 articles for eligibility. 23 articles were eligible which included 2258 children with an age range of 3.3-6.5 years. All included trials were shown in Figure 1.

The studies mainly included 10 items in America (6 in USA) ( Burrows et al., 2014; Jones et al., 2016; Gao et al., 2019; Alhassan et al., 2012; Brian et al., 2017; Wasenius et al., 2018; Palmer et al., 2019; Derri et al., 2001., Deli et al., 2006), 8 in Europe(Ivonen et al., 2011; Bonvin et al., 2013; Moher et al., 2015; Gao et al., 2019; Rocha et al., 2017; Lemos et al., 2012; Battaglia et al., 2019; Mostafavi et al., 2013; Yang, 2017), 3 in Asia (Wu, 2015; Jones et al., 2011; Hacke et al., 2017), and 2 in Oceania (Higgins \& Green, 2008; Stodden et al., 2008). The diversified courses integrated with FMS was adopted in 7 documents (Higgins \& Green, 2008; Alhassan et al., 2012; Palmer et al., 2019; Derri et al., 2001; Deli et al., 2006; Wu 2015; Stodden et al., 2008), 2 studies used object control program (Burrows et al., 2014; Palmer et al., 2019), and 12 reported the intervention which was performed by physical education teachers or trained staff (Bonvin et al., 2013; Higgins \& Green, 2008; Gao et al., 2019; Alhassan et al., 2012; Palmer et al., 2019; Derri et al., 2001; Deli et al., 2006; Lemos et al., 2012; Mostafavi et al., 2013; Yang, 2017; Wu, 2015; Stodden et al., 2008). Besides, 18 studies used TGMD to test (Burrows et al., 2014; Jones et al., 2016; Higgins \& Green, 2008; Gao et al., 2019; Alhassan et al., 2012; Brian et al., 2017; Wasenius et al., 2018; Palmer et al., 2019; Derri et al., 2001; Deli et al., 2006; Rocha et al., 2017; Lemos et al.,

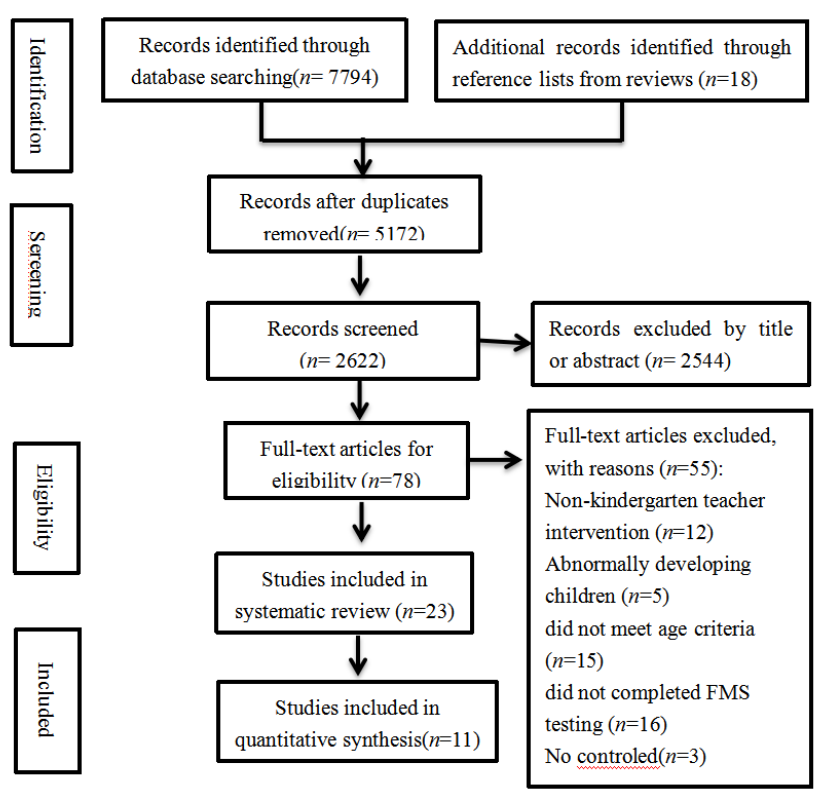

Figure 1. Flow diagram of study selection.
2012; Battaglia et al., 2019; Mostafavi et al., 2013; Wu S. 2015; Jones et al., 2011; Hacke et al., 2017; Stodden et al., 2008), and Zurich Neuromotor Assessment (ZNA) test (Moher et al., 2015), test of motor competence (KTK) (Ivonen et al., 2011), The APM Inventory (Bonvin et al., 2013), and Italian version of gross motor development test (Yang, 2017) respectively tested one item. In these studies, the shortest duration of intervention was five weeks (Deli et al., 2006), the longest was eighty weeks (Deli et al., 2006). The duration was shorter than or equal to ten weeks in 7 studies(Burrows et al., 2014; Alhassan et al., 2012; Brian et al., 2017; Deli et al., 2006; Rocha et al., 2017; Lemos et al., 2012; $\mathrm{Wu}, 2015)$, longer than ten weeks but shorter than or equal to twenty weeks in 6 documents (Ivonen et al., 2011; Jones et al., 2016; Battaglia et al., 2019; Yang, 2017; Jones et al., 2011; Stodden et al., 2008), and that of 9 studies was longer than twenty weeks (Bonvin et al., 2013; Higgins \& Green, 2008; Gao et al., 2019; Wasenius et al., 2018; Palmer et al., 2019; Derri et al., 2001; Mostafavi et al., 2013; Hacke et al., 2017). Moreover, the intervention of 8 studies was twice per weak (Burrows et al., 2014; Bonvin et al., 2013; Palmer et al., 2019; Rocha et al., 2017; Lemos et al., 2012; Battaglia et al., 2019; Yang, 2017; Jones et al., 2011), three times per week for 5 studies (Higgins \& Green, 2008; Alhassan et al., 2012; Deli et al., 2006; Wu, 2015; Stodden et al., 2008), and five times per week for 4 studies (Jones et al., 2016; Brian et al., 2017; Wasenius et al., 2018; Hacke et al., 2017). Most intervention included the time lasting for 20-60 minutes, and all interventions were conducted in kindergartens. The basic characteristics of the included articles were shown in Table 1.

\subsection{Quality evaluation}

The results of methodological quality (as shown in Figure 2) showed that there were 7 level A studies, 11 level B studies, and 5 level $\mathrm{C}$ studies. Among the 23 studies, 12 studies were randomly allocated by a computer (Ivonen et al., 2011; Jones et al., 2016; Moher et al., 2015; Gao et al., 2019; Wasenius et al., 2018; Derri et al., 2001; Deli et al., 2006; Rocha et al., 2017; Lemos et al., 2012; Mostafavi et al., 2013; Yang, 2017; Stodden et al., 2008). 6 studies used orderly and opaque sealed envelopes for distribution and hiding (Jones et al., 2016; Gao et al., 2019; Palmer et al., 2019; Derri et al., 2001; Lemos et al., 2012; Stodden et al., 2008). Besides, 6 studies was adopted double-blind for participants and implementers(Higgins \& Green, 2008; Palmer et al., 2019; Derri et al., 2001; Deli et al., 2006; Rocha et al., 2017; Lemos et al., 2012), and test evaluators were blinded in 6 studies(Jones et al., 2016; Higgins \& Green, 2008; Wasenius et al., 2018; Rocha et al., 2017; Hacke et al., 2017; Stodden et al., 2008), and other 6 studies did not report (Moher et al., 2015; Alhassan et al., 2012; Palmer et al., 2019; Battaglia et al., 2019; Mostafavi et al., 2013; Yang, 2017).

Besides, 11 studies were at the risk of measurement bias and there was subject dropout in 4 studies (Burrows et al., 2014; Bonvin et al., 2013; Rocha et al., 2017; Lemos et al., 2012). Other 2 studies only provided the score of sub item skill of mobile movement, while the total score was missing (Rocha et al., 2017; Lemos et al., 2012). In 3 studies, only partial FMS indicators were selectively tested (Higgins \& Green, 2008; Brian et al., 2017; Hacke et al., 2017), and it was not possible to merge the data. 
Table 1. Basic information of included studies.

\begin{tabular}{|c|c|c|c|c|c|}
\hline $\begin{array}{l}\text { Name, Year, } \\
\text { Country }\end{array}$ & Studydesign & $\begin{array}{c}\text { Sample characteristics, } \\
\text { Intervenor }\end{array}$ & $\begin{array}{l}\text { Intervention content, Methods and } \\
\text { means }\end{array}$ & $\begin{array}{l}\text { Evaluation tools } \\
\text { and indicators }\end{array}$ & Intervention results \\
\hline $\begin{array}{l}\text { Burrows et al. } \\
\text { (2014), Canada }\end{array}$ & $\mathrm{QE}$ & $\begin{array}{c}\mathrm{n}=40, \mathrm{INT}=15 \\
\mathrm{CON}=25, \mathrm{M}=6.5 \\
\text { Non-physical teachers }\end{array}$ & $\begin{array}{l}11 \text { weeks, } 60 \mathrm{~min} / \text { time, } 5 \text { times/week. } \\
\text { INT: Structured sports activities. CON: } \\
\text { Non-structural free activities. }\end{array}$ & $\begin{array}{l}\text { TGMD-2(FMS, } \\
\text { LMS, OCS) }\end{array}$ & $\begin{array}{l}\text { FMS:INT } \approx \mathrm{CON} \\
\text { LMS:INT } \approx \mathrm{CON} \\
\text { COS:INT } \approx \mathrm{CON}\end{array}$ \\
\hline $\begin{array}{l}\text { Adamo et al. } \\
\text { (2016), Canada }\end{array}$ & CRCT & $\begin{array}{c}\mathrm{n}=83, \mathrm{INT}=40 \\
\mathrm{CON}=43, \mathrm{M}=4.2, \\
\text { Trained teachers }\end{array}$ & $\begin{array}{l}24 \text { weeks. INT: Combine structured } \\
\text { and non-structured sports activities, } \\
\text { use skipping rope, ball games and other } \\
\text { equipment to practice movement skills, } \\
\text { and create a good learning environment. } \\
\text { CON: Non-structured sports activities. }\end{array}$ & $\begin{array}{l}\text { TGMD-2(FMS, } \\
\text { LMS, OCS) }\end{array}$ & $\begin{array}{l}\text { FMS:INT }>\text { CON } \\
\text { LMS:INT > CON } \\
\text { OCS:INT } \approx \text { CON }\end{array}$ \\
\hline $\begin{array}{l}\text { Bonvin et al. } \\
\quad(2013) \text {, } \\
\text { Switzerland }\end{array}$ & CRCT & $\begin{array}{c}\mathrm{n}=313, \mathrm{INT}=187, \\
\mathrm{CON}=201, \mathrm{M}=3.3, \\
\text { Non-physical teachers }\end{array}$ & $\begin{array}{l}\text { INT: The government provides certain } \\
\text { financial support to incorporate sports } \\
\text { activities into the daily lives of children, } \\
\text { but the government does not strictly } \\
\text { require the curriculum and frequency } \\
\text { of school sports activities. CON: } \\
\text { Non-structured sports activities. }\end{array}$ & $\mathrm{ZNA}$ & $\mathrm{INT} \approx \mathrm{CON}$ \\
\hline $\begin{array}{l}\text { Derri et al. (2001), } \\
\text { Greece }\end{array}$ & RCT & $\begin{array}{c}\mathrm{n}=68, \mathrm{INT}=35 \\
\mathrm{CON}=33, \mathrm{M}=5.43 \\
\text { Non-physical teachers }\end{array}$ & $\begin{array}{l}10 \text { weeks, } 35-40 \mathrm{~min} / \text { time, } 2 \text { times/ } \\
\text { week. INT: Participate in structured } \\
\text { sports activities, strengthen movement } \\
\text { development, combine music and } \\
\text { movement practice, CON: Participate in } \\
\text { non-structured sports activities, }\end{array}$ & TGMD-2(LMS) & LMS:INT > CON \\
\hline $\begin{array}{l}\text { Robinson et al. } \\
\text { (2016), United } \\
\text { States }\end{array}$ & RCT & $\begin{array}{c}\mathrm{n}=113, \mathrm{INT}=68 \\
\mathrm{CON}=45, \mathrm{M}=4.5 \\
\text { Trained teachers }\end{array}$ & $\begin{array}{l}5 \text { weeks, } 40 \mathrm{~min} / \text { time, } 3 \text { times/ } \\
\text { week. INT: Participate in CHAMP } \\
\text { structured courses. CON: Participate in } \\
\text { non-structured outdoor free activities. }\end{array}$ & $\begin{array}{l}\text { TGMD-2(FMS, } \\
\text { LMS, OCS) }\end{array}$ & $\begin{array}{l}\text { FMS:INT > CON } \\
\text { LMS:INT > CON } \\
\text { OCS:INT > CON }\end{array}$ \\
\hline $\begin{array}{c}\text { Gao et al. (2019), } \\
\text { United States }\end{array}$ & CT & $\begin{array}{c}\mathrm{n}=56, \mathrm{INT}=36 \\
\mathrm{CON}=20, \mathrm{M}=4.46, \text { Non- } \\
\text { physical teachers }\end{array}$ & $\begin{array}{l}8 \text { weeks, } 20 \mathrm{~min} / \text { time, } 5 \text { times/week. INT: } \\
\text { The intervention content is a structured } \\
\text { electronic video sports game, focusing } \\
\text { on the development of movements and } \\
\text { the cooperative practice of double and } \\
\text { multiplayer. CON: Participate in non- } \\
\text { structural outdoor sports activities and } \\
\text { play freely in the playground. }\end{array}$ & TGMD-2(FMS) & $\mathrm{INT} \approx \mathrm{CON}$ \\
\hline $\begin{array}{l}\text { Alhassan et al. } \\
\text { (2012), United } \\
\text { States }\end{array}$ & RCT & $\begin{aligned} & \mathrm{n}=71, \mathrm{INT}=43 \\
& \mathrm{CON}=28, \mathrm{M}=4.3, \text { Non- } \\
& \text { physical teachers }\end{aligned}$ & $\begin{array}{l}24 \text { weeks, } 30 \text { min/time, } 5 \text { times/week. } \\
\text { INT: Structured LMS-AP course, } 30 \\
\text { course plans, 3-5 minutes of music } \\
\text { warm-up, } 20 \text { minutes of FMS practice, } \\
5 \text { minutes of free performance, and } \\
\text { enhanced FMS. CON: Free play through } \\
\text { exercise equipment. }\end{array}$ & TGMD-2(LMS) & $\begin{array}{l}\text { LMS:INT } \approx \text { COM } \\
\text { LEAPING:INT > } \\
\text { CON }\end{array}$ \\
\hline $\begin{array}{c}\text { Deli et al. (2006), } \\
\text { Greece }\end{array}$ & CT & $\begin{array}{c}\mathrm{n}=75, \mathrm{INT} 1=25, \\
\mathrm{INT} 2=25, \mathrm{CON}=25, \\
\mathrm{M}=5.4, \text { Trained teachers }\end{array}$ & $\begin{array}{l}10 \text { weeks, } 35 \mathrm{~min} / \text { time, } 2 \text { times/week. } \\
\text { INT: Experimental group A participates in } \\
\text { structured sports, and experimental group } \\
\text { B consists of music plus structured sports. } \\
\text { CON: Non-structural free activities. }\end{array}$ & TGMD-2(LMS) & $\mathrm{INT}>\mathrm{CON}$ \\
\hline $\begin{array}{l}\text { Rocha et al. } \\
\text { (2017), Portugal }\end{array}$ & RCT & $\begin{array}{c}\mathrm{n}=33, \mathrm{INT} 1=11 \\
\mathrm{INT} 2=11, \mathrm{CON}=11 \\
\mathrm{M}=4.8, \text { Non-physical } \\
\text { teachers }\end{array}$ & $\begin{array}{l}20 \text { weeks, } 45 \text { min /time, } 2 \text { times/ } \\
\text { week. INT: Intervention by structured } \\
\text { physical activities. Experimental group } \\
1 \text { uses swimming, which includes water } \\
\text { exercises and basic water exercises. } \\
\text { Experimental group } 2 \text { uses football, } \\
\text { which includes the basic skills of } \\
\text { dribbling, passing, shooting and } \\
\text { the training of communication and } \\
\text { cooperation skills. CON: Non-structured } \\
\text { sports activities, mainly free play. }\end{array}$ & TGMD-2 & $\begin{array}{c}\text { soccer VS CON } \\
\text { LMS:INT > CON } \\
\text { OCS:INT > CON } \\
\text { swim VS CON } \\
\text { LSM:INT > CON } \\
\text { OCS:INT > CON } \\
\text { soccer VS swim } \\
\text { LMS:soccer > swim } \\
\text { OCS:soccer > swim }\end{array}$ \\
\hline
\end{tabular}

TGMD = gross motor development measurement; INT = the experimental group; CON = the control group; FMS = the basic motor skills; LMS = the movement skills; OCS = the object control skills; $\mathrm{CT}=$ the controlled trial; $\mathrm{RCT}=$ the randomized controlled trial; $\mathrm{CRCT}=$ the whole Group randomized controlled trials; $\mathrm{QE}=$ the quasi-trial design. 
Table 1. Continued...

\begin{tabular}{|c|c|c|c|c|c|}
\hline $\begin{array}{l}\text { Name, Year, } \\
\text { Country }\end{array}$ & Studydesign & $\begin{array}{c}\text { Sample characteristics, } \\
\text { Intervenor }\end{array}$ & $\begin{array}{l}\text { Intervention content, Methods and } \\
\text { means }\end{array}$ & $\begin{array}{l}\text { Evaluation tools } \\
\text { and indicators }\end{array}$ & Intervention results \\
\hline $\begin{array}{l}\text { Tortella et al. } \\
(2016) \text {, Italy }\end{array}$ & CT & $\begin{array}{c}\mathrm{n}=110, \mathrm{INT}=71 \\
\mathrm{CON}=39, \mathrm{M}=5.7, \text { Non- } \\
\text { physical teachers }\end{array}$ & $\begin{array}{l}20 \text { weeks, } 60 \mathrm{~min} / \text { time, } 1 \text { time/week. } \\
\text { INT: } 30 \text { minutes of structured exercise } \\
\text { plus } 30 \text { minutes of free activities. Under } \\
\text { the guidance of the teacher, you can } \\
\text { practice climbing, balance and other } \\
\text { physical qualities through various } \\
\text { equipment in the amusement park. } \\
\text { CON: Mainly non-structured activities. }\end{array}$ & $\begin{array}{c}\text { test of motor } \\
\text { competence KTK }\end{array}$ & FMS:INT > CON. \\
\hline $\begin{array}{l}\text { Lemos et al. } \\
\text { (2012), Brazil }\end{array}$ & RCT & $\begin{array}{l}\mathrm{n}=50, \mathrm{INT}=25 \\
\mathrm{CON}=25, \mathrm{M}=4.7 \\
\text { Trained teachers }\end{array}$ & $\begin{array}{l}32 \text { weeks, } 50 \mathrm{~min} / \text { time, } 1 \text { time/week. } \\
\text { INT: Structured physical education, } \\
\text { including cooperative games and } \\
\text { exercises in action skills in a fun } \\
\text { environment. CON: Free entertainment } \\
\text { activities in the playground under the } \\
\text { supervision of the head teacher. }\end{array}$ & $\begin{array}{c}\text { TGMD- } \\
\text { 2(LMS, OCS) }\end{array}$ & $\begin{array}{l}\text { LMS:INT > CON } \\
\text { OCS:INT > CON }\end{array}$ \\
\hline $\begin{array}{l}\text { Veldman et al. } \\
\text { (2017), United } \\
\text { States }\end{array}$ & $\mathrm{RCT}$ & $\begin{array}{c}\mathrm{n}=54, \mathrm{INT}=38 \\
\mathrm{CON}=16, \mathrm{M}=5.4 \\
\text { Non-physical teachers }\end{array}$ & $\begin{array}{l}9 \text { weeks, } 30 \mathrm{~min} / \text { time, } 2 \text { times/week. } \\
\text { INT: Participate in } 6 \text { sports (throwing, } \\
\text { receiving, serving, kicking, dribbling and } \\
\text { rolling). CON: Outdoor game activities. }\end{array}$ & TGMD-2(OCS) & OCS:INT > CON \\
\hline $\begin{array}{l}\text { Jones, et al. } \\
\text { (2011), Australia }\end{array}$ & CRCT & $\begin{array}{c}\mathrm{n}=97, \mathrm{INT}=52 \\
\mathrm{CON}=45, \mathrm{M}=4.8 \\
\text { Physical Education Teacher }\end{array}$ & $\begin{array}{l}20 \text { weeks, } 20 \mathrm{~min} / \text { time, } 3 \text { times/week. } \\
\text { INT: Jump Start structured courses, } \\
\text { mainly focus on children's movement } \\
\text { development, encourage children to } \\
\text { actively explore and try different ways of } \\
\text { movement skills exercise. CON: Regular } \\
\text { activities + games. }\end{array}$ & $\begin{array}{c}\text { TGMD- } \\
\text { 2(LMS, OCS) }\end{array}$ & $\begin{array}{l}\text { LMS:INT > OCS } \\
\text { OCS:INT > OCS }\end{array}$ \\
\hline $\begin{array}{l}\text { Jones et al. (2015), } \\
\text { Australia }\end{array}$ & RCT & $\begin{array}{l}\mathrm{n}=150, \mathrm{INT}=77 \\
\mathrm{CON}=73, \text { Trained } \\
\text { teachers }\end{array}$ & $\begin{array}{l}24 \text { weeks, } 20 \mathrm{~min} / \text { time, } 3 \text { times/week. } \\
\text { INT: JUNP START structured courses, } \\
\text { interesting methods, guide children } \\
\text { to practice various motor skills. CON: } \\
\text { Regular activities + games. }\end{array}$ & $\begin{array}{c}\text { TGMD- } \\
\text { 2(LMS, OCS) }\end{array}$ & $\begin{array}{l}\text { LMS:INT } \approx \text { OCS } \\
\text { COS:INT } \approx \text { OCS }\end{array}$ \\
\hline $\begin{array}{l}\text { Brian et al. (2017), } \\
\text { United States }\end{array}$ & QE & $\begin{array}{l}\mathrm{n}=57, \mathrm{INT}=26 \\
\mathrm{CON}=31, \mathrm{M}=4.8 \\
\text { Trained teachers }\end{array}$ & $\begin{array}{l}24 \text { weeks, } 30 \text { min/time, } 2 \text { times/week. } \\
\text { INT: SKIP structured course that } \\
\text { emphasizes movement development, } 2-3 \\
\text { minutes of music warm-up, } 2 \text { 10-minute } \\
\text { task practice of object control skills, } \\
\text { 3-4 minutes of stretching. CON: Non- } \\
\text { structural free activities. }\end{array}$ & TGMD-2(OCS) & OCS:INT > CON \\
\hline $\begin{array}{l}\text { Iivonen (2011), } \\
\text { Finland }\end{array}$ & CT & $\begin{array}{l}\mathrm{n}=84, \mathrm{INT}=39 \\
\mathrm{CON}=45, \mathrm{M}=4.8 \\
\text { Trained teachers }\end{array}$ & $\begin{array}{l}32 \text { weeks, } 45 \text { min/time, } 2 \text { times/week. } \\
\text { INT: Structured Physical Education } \\
\text { Curriculum PEC, the European Union } \\
\text { Early Preschool Children's Sports } \\
\text { Program. CON: Unstructured gaming } \\
\text { activities. }\end{array}$ & APM(FMS) & FMS:INT > CON \\
\hline $\begin{array}{l}\text { Battaglia et al. } \\
\text { (2019), Italy }\end{array}$ & CT & $\begin{array}{c}\mathrm{n}=119, \mathrm{INT}=90 \\
\mathrm{CON}=29, \mathrm{M}=5.5 \\
\text { Physical Education Teacher }\end{array}$ & $\begin{array}{l}16 \text { weeks, } 45 \text { min/time, } 2 \text { times/week. } \\
\text { INT: Structured physical activity class } \\
\text { PEP, highlighting the development of } \\
\text { activity awareness, basic movement } \\
\text { and perception-sensory skills, and } \\
\text { stimulating participation motivation } \\
\text { through games. CON: Non-structural } \\
\text { physical activity. }\end{array}$ & $\begin{array}{l}\text { Italian version } \\
\text { of gross motor } \\
\text { development test }\end{array}$ & $\begin{array}{l}\text { LMS:INT > CON } \\
\text { OCS:INT > CON }\end{array}$ \\
\hline $\begin{array}{l}\text { Wasenius et al. } \\
\text { (2018), Canada }\end{array}$ & CRCT & $\begin{array}{l}\mathrm{n}=215, \mathrm{INT} 1=59 \\
\mathrm{CON}=62, \mathrm{INT} 2=94 \\
\text { Trained teachers }\end{array}$ & $\begin{array}{l}24 \text { weeks, } 60 \mathrm{~min} / \text { time, } 5 \text { times/week. } \\
\text { INT1: Structured ABC activity course, } \\
\text { INT2: Combination of ABC activity } \\
\text { course + family intervention. CON: } \\
\text { Non-structural physical activity. }\end{array}$ & $\begin{array}{c}\text { TGMD- } \\
\text { 2(LMS, OCS) }\end{array}$ & $\begin{array}{l}\text { FMS:INT } \approx \mathrm{CON} \\
\text { LMS:TIN }>\text { CON } \\
\text { OCS:INT } \approx \text { CON }\end{array}$ \\
\hline
\end{tabular}

TGMD = gross motor development measurement; INT = the experimental group; CON = the control group; FMS = the basic motor skills; LMS = the movement skills; OCS = the object control skills; $\mathrm{CT}=$ the controlled trial; $\mathrm{RCT}=$ the randomized controlled trial; $\mathrm{CRCT}=$ the whole Group randomized controlled trials; $\mathrm{QE}=$ the quasi-trial design. 
Table 1. Continued...

\begin{tabular}{|c|c|c|c|c|c|}
\hline $\begin{array}{l}\text { Name, Year, } \\
\text { Country }\end{array}$ & Studydesign & $\begin{array}{l}\text { Sample characteristics, } \\
\text { Intervenor }\end{array}$ & $\begin{array}{l}\text { Intervention content, Methods and } \\
\text { means }\end{array}$ & $\begin{array}{l}\text { Evaluation tools } \\
\text { and indicators }\end{array}$ & Intervention results \\
\hline $\begin{array}{l}\text { Palmer et al. } \\
\text { (2019), United } \\
\text { States }\end{array}$ & RCT & $\begin{array}{c}\mathrm{n}=102, \mathrm{INT}=64, \\
\mathrm{CON}=38, \mathrm{M}=5.2, \\
\text { Physical Education Teacher }\end{array}$ & $\begin{array}{l}5 \text { weeks, } 40 \text { min/time, } 3 \text { times/week. INT: } \\
\text { The structured CHAMP course focuses on } \\
\text { movement development. The basic structure } \\
\text { includes a 2-minute warm-up, 3-4 minutes } \\
\text { of motor skills station instruction, 32-35 } \\
\text { minutes of motor skills practice, and 2-3 } \\
\text { minutes of relaxation activities. CON: Free } \\
\text { physical activity on the regular playground. }\end{array}$ & $\begin{array}{c}\text { TGMD- } \\
\text { 3(LMS, OCS) }\end{array}$ & $\begin{array}{l}\text { LMS:INT > CON } \\
\text { OCS:INT > CON }\end{array}$ \\
\hline $\begin{array}{l}\text { Mostafavi et al. } \\
\text { (2013), Iran }\end{array}$ & CT & $\begin{array}{c}\mathrm{n}=90, \text { INT } 1=30 \\
\text { INT2 }=30, \text { CON }=30 \\
\text { Physical Education } \\
\text { Teacher }\end{array}$ & $\begin{array}{c}8 \text { weeks, } 3 \text { times/week. INT1: SPARK } \\
\text { structured curriculum, using a } \\
\text { combination of sports, play and } \\
\text { entertainment. INT2: Gymnastics } \\
\text { intervention group. CON: Free play } \\
\text { activities. }\end{array}$ & $\begin{array}{l}\text { TGMD-2(FMS, } \\
\text { LMS, OCS) }\end{array}$ & $\begin{array}{l}\text { spark VS CON } \\
\text { FMS:INT > CON } \\
\text { LMS:INT >CON } \\
\text { OCS:INT >CON } \\
\text { gym VS COM } \\
\text { FMS:INT } \approx \text { COM } \\
\text { LMS:INT } \approx \text { COM } \\
\text { OCS:INT } \approx \text { COM }\end{array}$ \\
\hline $\begin{array}{l}\text { Veldman et al. } \\
\text { (2016), Crolia }\end{array}$ & RCT & $\begin{array}{c}\mathrm{n}=78, \mathrm{INT} 1=21 \\
\mathrm{INT} 2=14, \mathrm{INT} 3=22, \\
\text { CON }=21, \mathrm{M}=5.3, \text { Non- } \\
\text { physical teachers }\end{array}$ & $\begin{array}{l}80 \text { weeks, } 45 \text { min/week, } 2 \text { times/week. } \\
\text { INT1: Use mixed sports interventions } \\
\text { (football, volleyball, handball, } \\
\text { badminton, tennis, gymnastics, } \\
\text { martial arts, etc.), INT2: use rhythmic } \\
\text { gymnastics, including balance, flexibility } \\
\text { and dance, etc., INT3: football, including } \\
\text { basic skills and competitions. CON: } \\
\text { School sports activities arranged daily. }\end{array}$ & $\begin{array}{c}\text { TGMD- } \\
\text { 2(LMS, OCS) }\end{array}$ & $\begin{array}{l}\text { Mixed intervention } \\
\text { VS football and } \\
\text { aerobics LMS:INT } \\
\text { > CON OCS:INT } \\
\text { > CON Football, } \\
\text { aerobics and regular } \\
\quad \text { activities } \\
\text { LMS:INT } \approx \text { CON } \\
\text { OCS:INT } \approx \text { CON }\end{array}$ \\
\hline Wu (2015), China & RCT & $\begin{aligned} & \mathrm{n}=80, \mathrm{INT}=40 \\
& \mathrm{CON}=40, \mathrm{M}=4.5, \text { Non- } \\
& \text { physical teachers }\end{aligned}$ & $\begin{array}{l}48 \text { weeks, } 20-30 \mathrm{~min} / \text { time, } 5 \text { times/ } \\
\text { week. INT: Rhythmic physical activity } \\
\text { that integrates fundamental movement } \\
\text { development, with music. CON: Free } \\
\text { activities. }\end{array}$ & $\begin{array}{l}\text { TGMD-2(FMS, } \\
\text { LMS, OCS) }\end{array}$ & $\begin{array}{l}\text { FMS:INT > CON } \\
\text { LMS:INT > CON } \\
\text { OCS:INT > CON }\end{array}$ \\
\hline $\begin{array}{l}\text { Yang (2017), } \\
\text { China }\end{array}$ & RCT & $\begin{array}{c}\mathrm{n}=120, \mathrm{INT}=60 \\
\mathrm{CON}=60, \mathrm{M}=5.7, \text { Non- } \\
\text { physical teachers }\end{array}$ & $\begin{array}{l}12 \text { weeks, } 30-40 \mathrm{~min} / \text { time, } 2 \text { times/ } \\
\text { week. INT: Structural sports from the } \\
\text { perspective of movement development. } \\
\text { CON: Free activities. }\end{array}$ & $\begin{array}{l}\text { TGMD-3(FMS, } \\
\text { LMS, OCS) }\end{array}$ & $\begin{array}{l}\text { FMS:INT > CON } \\
\text { LMS:INT > CON } \\
\text { OCS:INT > CON }\end{array}$ \\
\hline
\end{tabular}

TGMD = gross motor development measurement; INT = the experimental group; $\mathrm{CON}=$ the control group; FMS = the basic motor skills; LMS = the movement skills; OCS = the object control skills; $\mathrm{CT}=$ the controlled trial; $\mathrm{RCT}=$ the randomized controlled trial; $\mathrm{CRCT}=$ the whole Group randomized controlled trials; $\mathrm{QE}=$ the quasi-trial design .

\begin{tabular}{|c|c|c|c|c|c|c|c|c|c|c|c|c|c|c|c|c|c|c|c|c|c|c|c|}
\hline 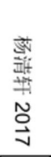 & 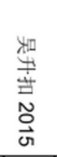 & $\begin{array}{l}\tilde{N} \\
\tilde{J} \\
0 \\
0 \\
\tilde{O} \\
0 \\
0\end{array}$ & 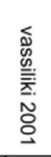 & 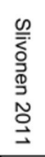 & 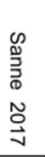 & 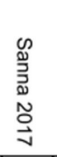 & 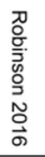 & $\begin{array}{l}\mathbb{D} \\
\mathbb{N} \\
N \\
\stackrel{O}{\omega}\end{array}$ & 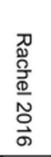 & $\begin{array}{l}\bar{D} \\
\stackrel{\tilde{J}}{\vec{N}} \\
\overrightarrow{\tilde{N}} \\
\tilde{N} \\
\stackrel{0}{0}\end{array}$ & $\begin{array}{l}\underline{z} \\
\text { zi } \\
\tilde{N} \\
\stackrel{0}{\infty}\end{array}$ & 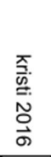 & 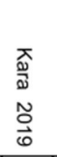 & 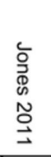 & $\begin{array}{l}I \\
\frac{I}{\Phi} \\
\mathbb{D} \\
\mathbb{J} \\
\tilde{O} \\
\mathbb{J}\end{array}$ & 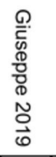 & 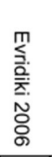 & 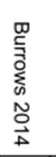 & 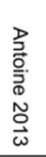 & 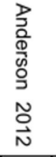 & 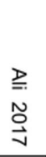 & 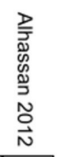 & \\
\hline 0 & O & 0 & $\odot$ & 8 & (1) & O & (1) & 0 & 0 & + & + & + & + & $\odot$ & O & + & $\odot$ & $\odot$ & + & + & (1) & + & Random sequence generation (selection bias) \\
\hline 0 & 0 & 0 & (1) & 0 & 0 & 0 & (1) & 0 & 0 & (1) & + & + & 0 & + & 0 & 0 & + & + & 0 & $\sim$ & + & 0 & Allocation concealment (selection bias) \\
\hline 0 & $\sim$ & 0 & + & 0 & (1) & 0 & (1) & 0 & + & (1) & + & 0 & + & 0 & 0 & 0 & + & 0 & 0 & 0 & + & $\omega$ & Blinding of participants and personnel (performance bias) \\
\hline O & + & O & $\odot$ & 8 & O & O & $\sim$ & O & $\odot$ & (1) & (1) & O & O & $\odot$ & $\omega$ & $\sim$ & O & + & $\sim$ & $\sim$ & $\sim$ & + & Blinding of outcome assessment (detection bias) \\
\hline+ & + & $\odot$ & (1) & (1) & $\odot$ & (1) & + & $\sim$ & $\sim$ & + & $\odot$ & + & + & $\odot$ & + & $\sim$ & (1) & $\sim$ & + & + & $\odot$ & + & Incomplete outcome data (attrition bias) \\
\hline+ & $\sim$ & O & (1) & O & $\odot$ & + & + & + & O & + & $\odot$ & 0 & $\sim$ & O & $\sim$ & O & O & + & O & + & $\odot$ & + & Selective reporting (reporting bias) \\
\hline+ & + & (1) & $\odot$ & $\odot$ & O & (1) & (1) & (1) & (1) & + & (1) & + & (1) & + & (1) & + & O & (1) & $\sim$ & (1) & + & + & Other bias \\
\hline
\end{tabular}

Figure 2. A schematic of quality evaluation based on Cochrane methodology.

In addition, 12 papers have other risk of bias due to sample size less than 30(Burrows et al., 2014; Jones et al., 2016; Higgins \& Green, 2008; Alhassan et al., 2012; Brian et al., 2017; Derri et al.,
2001; Deli et al., 2006; Lemos et al., 2012; Battaglia et al., 2019; Mostafavi et al., 2013; Wu, 2015). Most studies have detailed description of the intervention content, organization form and 
training plan of the experimental group, but lack of detailed description of the intervention method, content and form of the control group.

\subsection{Effect of intervention on FMS}

Among the 23 included studies, we described 12 of them only qualitatively since the data in these studies did not meet the requirements (Table 2). Among them, 6 studies pointed out that compared with free activity, structured sports activities have a significant role in promoting the development of FMS in preschool children (Ivonen et al., 2011; Bonvin et al., 2013; Rocha et al., 2017; Lemos et al., 2012; Yang, 2017; Wu, 2015; Stodden et al., 2008). Besides, the results of 3 studies showed that the difference between sports and free activity after intervention did not have statistical significance (Moher et al., 2015; Higgins \& Green, 2008; Brian et al., 2017). 2 research results showed that the sports activities only promoted the basic movement skills (Gao et al., 2019; Wasenius et al., 2018). Moreover, the left 1 study conducted comparative intervention through diversified SPARK curriculum, gymnastics and free exercise and showed significant differences between the diversified SPARK course group and the single gymnastics and free exercise group, while the difference between gymnastics and free exercise was not significant (Wu, 2015).

Meta-analysis was carried out on 11 eligible studies and the overall FMS, LMS and OCS forest maps were drawn in Figure 3. The results of 5 meta-analysis (Alhassan et al., 2012; Derri et al., 2001; Deli et al., 2006; Jones et al., 2011; Hacke et al., 2017) showed that there was a significant difference in the impact of sports intervention and free activity on the overall FMS (SMD $\left.=1.50,95 \% \mathrm{Cl}[1.29,1.71], I^{2}=89.7 \%\right)$ (Figure 3A). In the summary of FMS subscale, 8 meta-analysis (Jones et al., 2016; Alhassan et al., 2012; Derri et al., 2001; Deli et al., 2006;
Battaglia et al., 2019; Mostafavi et al., 2013; Jones et al., 2011; Hacke et al., 2017) results showed that compared with free exercise, the influence of physical activity intervention on LMS in preschool children was statistically significant $(\mathrm{SMD}=1.56$, $95 \% \mathrm{Cl}[1.38,1.75], I^{2}=59.2 \%$ ) (Figure 3B). The results of 10 meta-analysis (Burrows et al., 2014; Jones et al., 2016; Alhassan et al., 2012; Palmer et al., 2019; Derri et al., 2001; Deli et al., 2006; Battaglia et al., 2019; Mostafavi et al., 2013; Jones et al., 2011; Hacke et al., 2017) showed a significant difference between physical activity intervention and free activity on OCS in preschool children (SMD $=1.43,95 \% \mathrm{Cl}[1.25,1.61]$, $\mathrm{I}^{2}=93.8 \%$ ) (Figure 3C). The results of the combination of overall FMS and OCS were significantly heterogeneous.

\subsection{Heterogeneity, subgroup and exploratory analysis}

In order to explore the source of heterogeneity, multivariate meta-regression analysis was carried out on the research characteristics that may lead to heterogeneity. The results showed that (Table 3), in terms of LMS, the correlation of LMS with curriculum and teachers was statistically significant $(\mathrm{P}<0.05)$, while its correlation with project, intervention frequency, intervention cycle, intervention time was not significant $(\mathrm{P}>0.05)$. In terms of OCS, the correlation between project and OCS was very significant $(\mathrm{P}<0.01)$, while the correlation between curriculum, teachers, intervention dose and other factors was not significant $(P>0.05)$. Therefore, curriculum, teachers, projects and other factors may be the main causes of heterogeneity. In addition to the above factors, exercise intensity may also be the cause of heterogeneity, but it cannot be verified due to lack of effective data.

The results showed that after grouping, the heterogeneity of each group decreased to medium and low levels (Tables 4 and 5). In terms of LMS (Table 4), through subgroup analysis of

Table 2. Intervention characteristics of included studies.

\begin{tabular}{|c|c|c|c|c|c|}
\hline Content & Proportion (\%) & Effective rate (\%) & Content & Proportion (\%) & Effective rate (\%) \\
\hline $\begin{array}{l}\text { Intervention } \\
\text { duration }\end{array}$ & & & Course & & \\
\hline $20-30 \mathrm{~min}$ & 30 & 25 & Multi-courses integrated with FMS & 53 & 57 \\
\hline $30-45 \mathrm{~min}$ & 15 & 100 & Regular course & 46 & 33 \\
\hline $45-60 \mathrm{~min}$ & 30 & 100 & & & \\
\hline Not reported & 23 & & & & \\
\hline $\begin{array}{l}\text { Frequency } \\
\text { (times/week) }\end{array}$ & & & Teacher & & \\
\hline $1-3$ & 69 & 88 & $\begin{array}{c}\text { Physical education teachers or trained } \\
\text { teachers }\end{array}$ & 53 & 57 \\
\hline $4-5$ & 15 & 0 & Non-physical teachers & 46 & 50 \\
\hline Not reported & 15 & & & & \\
\hline Period (week) & & & Teaching content & & \\
\hline $5-10$ & 30 & 75 & Object control sports & 30 & 75 \\
\hline $10-20$ & 23 & 100 & Non-control sports & 69 & 44 \\
\hline $20>$ & 46 & 50 & & & \\
\hline
\end{tabular}

Proportion (\%): the proportion of the number in total 23 studies; Effective rate (\%): the proportion of intervention group significantly higher than that of control group in a certain classification standard. 

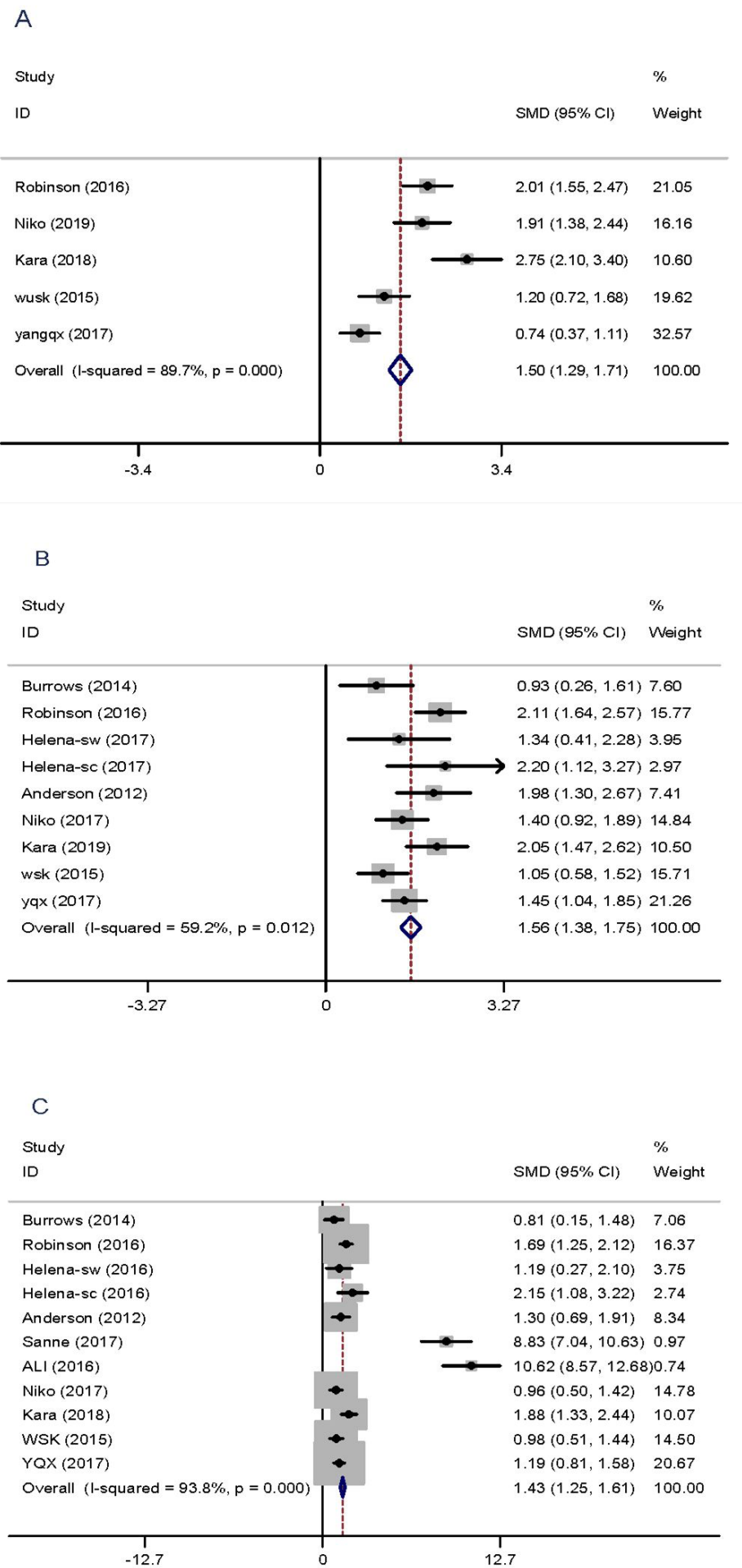

Figure 3. (A) the forest map of the impact of structural sports activities on the overall FMS; (B) the forest map of the impact of structural sports activities on LMS; (C) the forest map of the impact of structural sports activities on OCS.

curriculum and teachers, the heterogeneity of LMS has reduced to a low level, and it integrated into the multi-structure courses with FMS (SMD $=1.88,95 \%$ CI [1.62, $P<0.05], 2.14]$ ) was better than that of single structured activity course $(\mathrm{SMD}=1.23$, $95 \%$ CI $[0.96,1.50])$, and that of PE major or trained teachers $(\mathrm{SMD}=1.86,95 \% \mathrm{CI}[1.59,2.13])$ was better than that of ordinary teachers $(\mathrm{SMD}=1.29,95 \% \mathrm{CI}[1.03,1.54])$. In terms of OCS (Table 5), through subgroup analysis of project characteristics, the heterogeneity of each group decreased to a lower level, and the effect of equipment manipulation (SMD $=9.61,95 \%$ CI $[8.26,10.96])$ on OCS was significantly better than that of non-apparatus manipulation $(\mathrm{SMD}=1.29,95 \% \mathrm{CI}[1.11,1.47])$. 
Table 3. Results of multivariate meta-regression analysis

\begin{tabular}{|c|c|c|c|c|c|c|c|c|}
\hline & \multicolumn{4}{|c|}{ LMS } & \multicolumn{4}{|c|}{ OCS } \\
\hline & $\begin{array}{c}\text { Regression } \\
\text { coefficient }\end{array}$ & Standard error & $\mathrm{t}$ & $\mathrm{P}$ & $\begin{array}{c}\text { Regression } \\
\text { coefficient }\end{array}$ & Standard error & $\mathrm{t}$ & $\mathrm{P}$ \\
\hline Course & 0.676 & 0.227 & 2.98 & $0.021^{\star}$ & 0.291 & 0.708 & 0.41 & 0.702 \\
\hline Item & 0.648 & 0.674 & 0.96 & 0.368 & 8.068 & 0.788 & 10.24 & $0.001^{\star *}$ \\
\hline Teacher & 0.576 & 0.241 & 2.38 & $0.049^{*}$ & 0.074 & 0.751 & 0.1 & 0.926 \\
\hline Frequency & -0.631 & 0.289 & -2.18 & 0.081 & -0.489 & 0.484 & -1.01 & 0.369 \\
\hline Period & -0.093 & 0.174 & -0.53 & 0.616 & -0.033 & 0.279 & -0.12 & 0.911 \\
\hline Duration & 0.189 & 0.131 & 1.45 & 0.208 & -0.06 & 0.223 & -0.27 & 0.798 \\
\hline
\end{tabular}

${ }^{*}$ The difference was significant $(\mathrm{P}<0.05)$; ${ }^{*}$ The difference was very significant $(\mathrm{P}<0.01)$.

Table 4. LMS subgroup analysis.

\begin{tabular}{llcccccc}
\hline \multicolumn{1}{c}{ Grouping standard } & $\begin{array}{c}\text { Number of } \\
\text { literature }\end{array}$ & $Z$ & $P$ & $I^{2}(\%)$ & SMD & SMD 95\%cl \\
\hline Course & $\begin{array}{l}\text { Multi-structure courses } \\
\text { integrated with FMS } \\
\text { Single-structure activity }\end{array}$ & 5 & 9.05 & 0.25 & 25.8 & 1.88 & $1.62,2.14$ \\
& $\begin{array}{l}\text { courses } \\
\text { PE teacher / trained teacher }\end{array}$ & 4 & 14.23 & 0.479 & 0 & 1.23 & $0.96,1.50$ \\
\multirow{2}{*}{ Teacher } & Non-PE teacher & 5 & 13.68 & 0.169 & 40.4 & 1.86 \\
& & & 9.74 & 0.25 & 25.8 & 1.29 \\
\hline
\end{tabular}

Table 5. OCS subgroup analysis.

\begin{tabular}{|c|c|c|c|c|c|c|c|}
\hline & Grouping standard & $\begin{array}{c}\text { Number of } \\
\text { articles }\end{array}$ & $Z$ & $P$ & $I^{2}(\%)$ & SMD & SMD 95\%cl \\
\hline \multirow[t]{2}{*}{ Types of exercises } & Equipment manipulation exercises & 2 & 10.83 & 0.199 & 39.5 & 9.61 & $8.26,10.96$ \\
\hline & Non-apparatus manipulation exercises & 9 & 9.66 & 0.043 & 49.9 & 1.29 & $1.11,1.47$ \\
\hline
\end{tabular}

Table 6. Subgroup analysis.

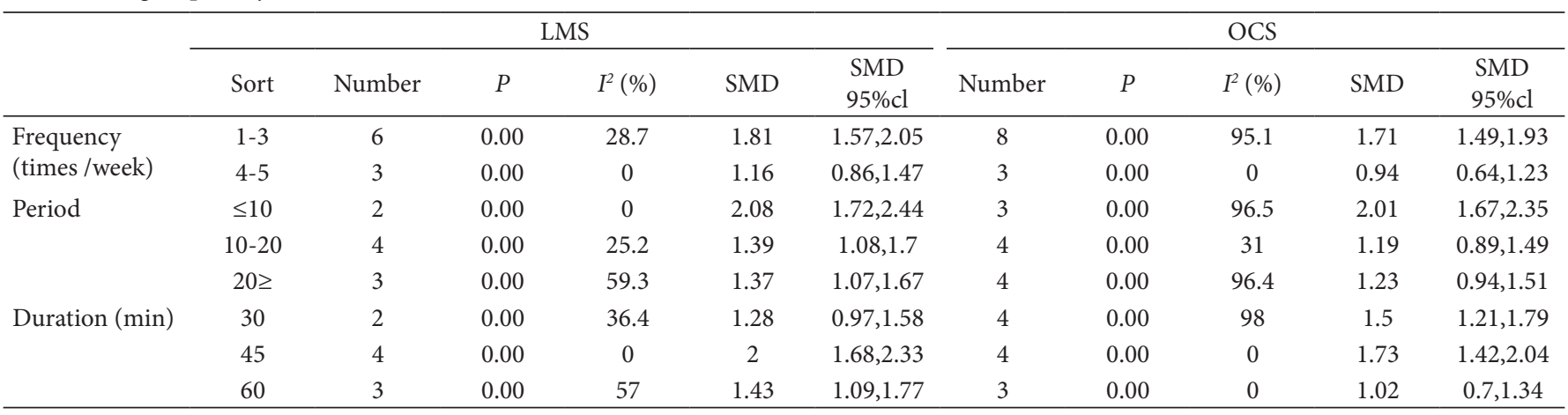

Hierarchical multi-level subgroup analysis showed that the intervention effect of each subgroup on FMS was statistically significant (Table 6). In terms of intervention frequency, 1-3 times a week $\left(\mathrm{SMD}_{\mathrm{LMS}}=1.81,95 \%[1.57,2.05], I^{2}=28.7 \%\right)$ had better effect on FMS than 4-5 times a week $\left(\mathrm{SMD}_{\mathrm{LMS}}=0.86\right.$, $\left.95 \%[0.86,1.47], I^{2}=0 \%\right)$. Regarding the training duration, 45 minutes $\left(\mathrm{SMD}_{\mathrm{LMS}}=2,95 \%[1.68,2.33], I^{2}=0 \%\right)$ were better than 30 minutes $\left(\mathrm{SMD}_{\mathrm{LMS}}=1.28,95 \%[0.97,1.58], I^{2}=36.4 \%\right)$ and 60 minutes $\left(\mathrm{SMD}_{\mathrm{LMS}}=1.43,95 \%[1.09,1.77], I^{2}=57 \%\right)$. In terms of intervention cycle, the intervention effect less than 10 weeks $\left(\mathrm{SMD}_{\mathrm{LMS}}=2.08,95 \%[1.72,2.44], I^{2}=0 \%\right)$ was better than that of 10 -20 weeks $\left(\mathrm{SMD}_{\mathrm{LMS}}=1.39,95 \%[1.08,1.7], I^{2}=25.2 \%\right)$ and more than 20 weeks $\left(\mathrm{SMD}_{\mathrm{LMS}}=1.37,95 \%\right.$ [1.07, 1.67], $\left.I^{2}=59.3 \%\right)$.

\subsection{Publication bias}

According to Begger's analysis (Figure 4), there was obvious asymmetry in small and medium sample of OCS studies (Figure 4A). The results show that $\mathrm{OCS}(\mathrm{t}=3.71, \mathrm{P}=0.005)$ had publication bias, and the difference was statistically significant. However, the symmetry of LMS funnel plot was apparent (Figure 4B), and the results showed no publication bias in $\mathrm{LMS}(\mathrm{t}=0.44, \mathrm{P}=0.67)$. 

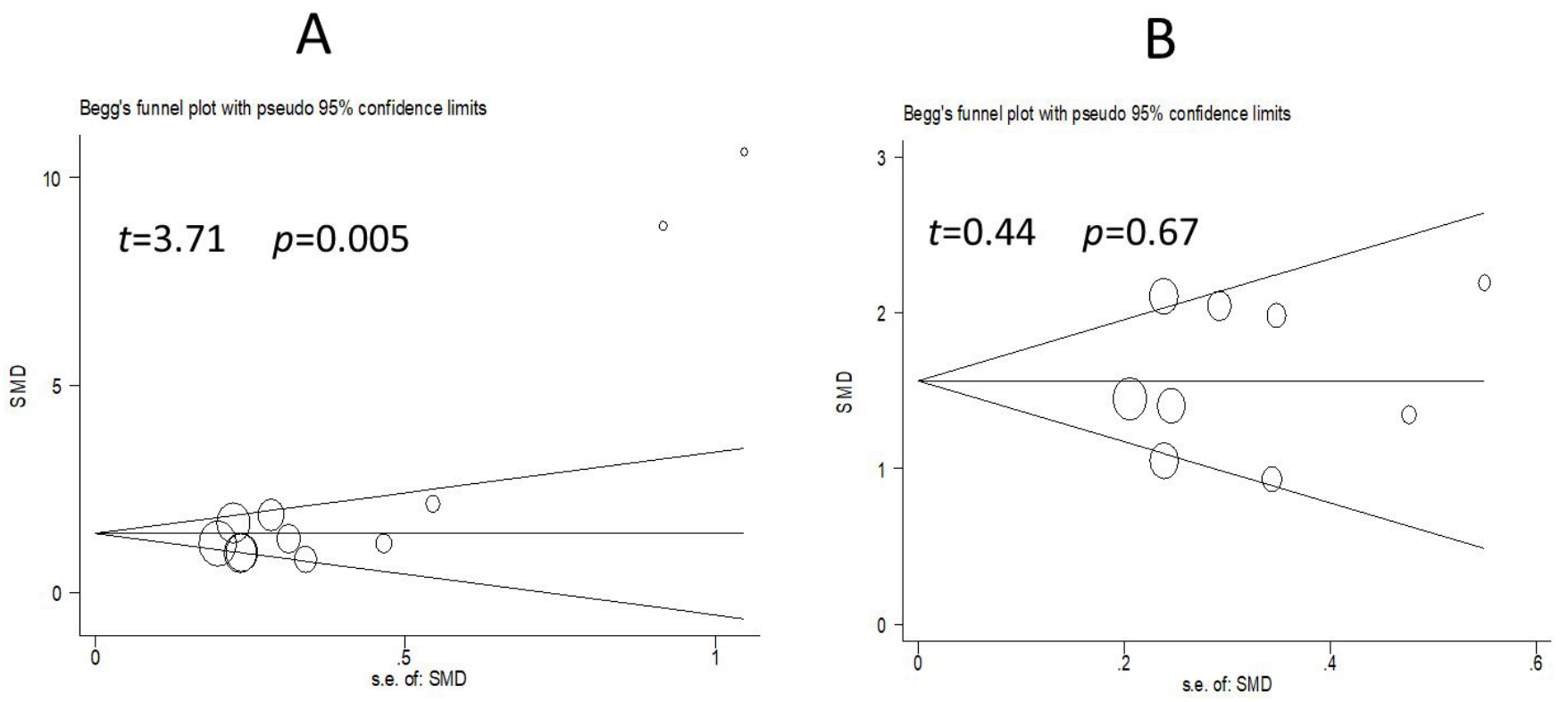

Figure 4. (A) shows the funnel diagram of OCS publication bias; (B) shows the funnel chart of LMS publication bias.

\section{Discussion}

In this study, preschool children with typical development were selected as the research objects. By participating in planned, purposeful and organized structural sports activities led by teachers, the comprehensive quantitative results showed that sports activities can obviously promote the overall FMS proficiency $(\mathrm{SMD}=1.50)$, mobile movement skills $(\mathrm{SMD}=1.56)$ and object control skills (SMD=1.43) of preschool children.

Stodden et al. (2008), Williams et al. (2008) and Ji (2019) proposed the development model of FMS and pointed out that there was a bidirectional interaction between FMS and physical activity, and physical fitness and body perception ability were the intermediate variables of bidirectional interaction (Van Capelle et al., 2017). Preschool children's physical activities are composed of teaching, learning and practice, etc. Therefore, teachers, courses, teaching methods, exercise intensity, exercise density and other important factors significantly affect the physical and mental health development of preschool children (Hamstra-Wright et al., 2006).

Capelle's research believes that physical activity intervention has a great influence on preschool children's object control skills $(\mathrm{SMD}=1.06)$, while it has a smaller influence on locomotor skills $(S M D=0.62)$ (Robinson et al., 2017). Our study showed that physical activity had a better effect on preschoolers' locomotor skills than their object control skills.

Karrie's research points out that boys benefit more from the physical activities on FMS than girls (Hung et al., 2017). Therefore, sports activities to improve FMS of preschool children may be affected by multiple factors such as gender and program. In this study, the intervention programs were compared in stratified subgroups and showed that combined equipment manipulation exercise ( $\mathrm{SMD}=9.61)$ had a significantly greater impact on OCS in children than non-appliance control exercise $(\mathrm{SMD}=1.29)$.
Relevant studies have also pointed out that ball games and programs of object control are more conducive to developing children's hand-eye, upper and lower limbs and whole body coordination ability, which are more beneficial for the development of children's OCS (Xin et al., 2019; Logan et al., 2012). Therefore, the gender ratio of participants in the study and the differences in the intervention programs may be the main reasons for the two different results of the studies. The research above also suggests that the adjustment of FMS by gender and sports program should be considered in the arrangement of children's physical activity.

Dose of physical education intervention is an important influence factor for physical activity to promote FMS development in preschool children (Cattuzzo et al., 2016). Logand et al. suggests that at least twice-weekly intervention can improve FMS for preschool children (Wick et al., 2017), while Maria believes that intervention should be at least three times a week and last more than 30 minutes every time in order to improve FMS (Lai et al., 2014). There is still lack of prescriptive basis regarding what kind of intervention dose is more beneficial to the development of preschool children's FMS. Our results show that structured physical activity for about 10 weeks, 1-3 times a week for 45 minutes each time, is more beneficial to FMS development in preschool children. The effect of physical activity intervention at less than 10 weeks $(\mathrm{SMD}=2.08)$ and 10 20 weeks ( $\mathrm{SMD}=1.39$ ) on FMS in preschool children was better than that at more than 20 weeks (SMD=1.37). Wick's study also believed that intervention lasting from 4 weeks to 5 months had a significantly better effect on FMS than the intervention lasting more than 6 months (Brian et al., 2017). Regarding the intervention does, our study show that the effect of physical activity on FMS of preschool children is not linearly related to the intervention does, it was consistent with Wick's conclusion.

Abbey et al. pointed out that the single intervention lasted more than 30 minutes every time, 4-5 times a week, and the 
intervention even in a higher frequency produced greater benefits for FMS (Robinson et al., 2017). Consistently, we showed that the training effect of $1-3$ times $(\mathrm{SMD}=1.81)$ per week was better than that of $4-5$ times $(\mathrm{SMD}=1.16)$. The training effect of 45 minutes $(\mathrm{SMD}=2.00)$ was better than that of 30 minutes $(\mathrm{SMD}=1.28)$ and 60 minutes $(\mathrm{SMD}=1.43)$. The effect of physical activity on FMS in preschool children does not improve with the increase of intervention cycle, frequency and duration of single intervention, which may be due to the following reasons: First of all, excessive intervention frequency may lead to children's compliance with physical activities, the novelty of teaching content and the interest of organizational methods are decreased, which weaken children's motivation to participate in physical activities, thus preschooler children lose their enthusiasm (Riethmuller, 2009). Secondly, the intervention with long period may be influenced by FMS proficiency, and the ceiling effect forms (Cattuzzo et al., 2016). Thirdly, because preschool children's attention is generally maintained for about 10-15 minutes, a too-long or too-short intervention may affect the development of FMS. As reported in many researches, the intervention is divided into warm-up, basic part and relaxation part, and the time for warm-up and relaxation part is generally controlled at about $10 \mathrm{~min}$ (Brusseau et al., 2018). If a single practice time lasts for 20-30 $\mathrm{min}$, the basic action practice time is only about 10 min, which may cause unfavorable to FMS development due to lack of exercise. However, if a single intervention lasts too long, it may cause cognitive decline of children due to fatigue, which is not conducive to the development of FMS (S H. 1980).

As an important part of structured physical activity, curriculum design is an important factor affecting children's participation in physical activity (Maluly et al., 2020; Barreto et al., 2018). Subgroup analysis shows that hybrid intervention courses are obviously superior to the first two courses (Xiong et al., 2017). We show that the multi-structure curriculum integrated with FMS ( $\mathrm{SMD}=1.88)$ is better than the single structure activity curriculum $(\mathrm{SMD}=1.23)$ in promoting children's FMS, and integrating fundamental movement skills is more beneficial to the development of preschool children's FMS, which is consistent with JANE's research results.

Our study has some limitations. The results are limited by study design and not completely consistent, such as CTs or RCTs with unclear randomization procedures and lack of information regarding allocation concealment. Our study only reviews articles of English and Chinese, which could be one of the reasons for publication bias. We suggest that the following study should pay more attention to the long-term effect of physical activity on FMS of preschool children, and not only consider the basic demographic characteristics, but also social factors.

\section{Conclusions and suggestions}

Sports activities have positive promotion value for preschool children's fundamental motor skills. Multi-structure courses with fundamental motor skills, equipment control sports and professional teachers are more conducive to the development of preschool children's fundamental motor skills. Structured physical activity intervention for 45 minutes every time, 1-3 times a week for 10 weeks, can effectively promote the development of preschool children's fundamental motor skills. We suggest that kindergartens should design fundamental motor skills development courses in a targeted way, to constantly improve the quality of children's physical activity teaching by supplementing professional sports teacher and training teacher, and develop children's fundamental motor skills, so as to promote the overall development of children's health level.

\section{Funding}

Shanghai Educational Science Research Project of 2020 (C22020030), General Project of Humanities and Social Sciences Foundation of Ministry of Education (18YJA890010).

\section{References}

Adamo, K. B., Wilson, S., Harvey, A. L. J., Grattan, K. P., Naylor, P. J., Temple, V. A., \& Goldfield, G. S. (2016). Does intervening in childcare settings impact fundamental movement skill development? Medicine and Science in Sports and Exercise, 48(5), 926-932. http://dx.doi. org/10.1249/MSS.0000000000000838. PMid:26656776.

Alhassan, S., Nwaokelemeh, O., Ghazarian, M., Roberts, J., Mendoza, A. \& Shitole, S. (2012). Effects of locomotor skill program on minority preschoolers' physical activity levels. Pediatric Exercise Science, 24(3), 435-449. http://dx.doi.org/10.1123/pes.24.3.435. PMid:22971559.

Barreto, C. M. L. G., Rangel, A., Urbano, S. A., Bezerra, J., \& Oliveira, C. (2018). Equine milk and its potential use in the human diet. Food Science and Technology, 39(12), 1-7.

Battaglia, G., Alesi, M., Tabacchi, G., Palma, A., \& Bellafiore, M. (2019). The development of motor and pre-literacy skills by a physical education program in preschool children: a non-randomized pilot trial. Frontiers in Psychology, 9, 2694. http://dx.doi.org/10.3389/ fpsyg.2018.02694. PMid:30687164.

Bonvin, A., Barral, J., Kakebeeke, T. H., Kriemler, S., Longchamp, A., Schindler, C., Marques-Vidal, P., \& Puder, J. J. (2013). Effect of a governmentally-led physical activity program on motor skills in young children attending child care centers: a cluster randomized controlled trial. The International Journal of Behavioral Nutrition and Physical Activity, 10(1), 90. http://dx.doi.org/10.1186/14795868-10-90. PMid:23835207.

Brian, A., Goodway, J. D., Logan, J. A., \& Sutherland, S. (2017). SKIPing with teachers: an early years motor skill intervention. Physical Education and Sport Pedagogy, 22(3), 270-282. http://dx.doi.org/1 0.1080/17408989.2016.1176133.

Brusseau, T. A., Hannon, J. C., Fu, Y., Fang, Y., Nam, K., Goodrum, S., \& Burns, R. D. (2018). Trends in physical activity, health-related fitness, and gross motor skills in children during a two-year comprehensive school physical activity program. Journal of Science and Medicine in Sport, 21(8), 828-832. http://dx.doi.org/10.1016/j.jsams.2017.12.015. PMid:29373204.

Bryant, E. S., Duncan, M. J., \& Birch, S. L. (2014). Fundamental movement skills and weight status in British primary school children. Oxfordshire: Taylor \& Francis.

Burrows, E. J., Keats, M. R., \& Kolen, A. M. (2014). Contributions of after school programs to the development of fundamental movement skills in children. International Journal of Exercise Science, 7(3), 236-249. PMid:27293501.

Cattuzzo, M. T., Henrique, R. S., Ré, A. H. N., Oliveira, I. S., Melo, B. M., Moura, M. S., Araújo, R. C., \& Stodden, D. (2016). Motor competence and health related physical fitness in youth: a systematic review. Journal of Science and Medicine in Sport, 19(2), 123-129. http://dx.doi.org/10.1016/j.jsams.2014.12.004. PMid:25554655. 
Cools, W., Martelaer, K. D., Samaey, C., \& Andries, C. (2009). Movement skill assessment of typically developing preschool children: a review of seven movement skill assessment tools. Journal of Sports Science \& Medicine, 8(2), 154-168. PMid:24149522.

D’Hondt, E., Deforche, B., Gentier, I., Bourdeaudhuij, I., Vaeyens, R., Philippaerts, R., \& Lenoir, M. (2013). A longitudinal analysis of gross motor coordination in overweight and obese children versus normal-weight peers. International Journal of Obesity, 37(1), 61-67. http://dx.doi.org/10.1038/ijo.2012.55. PMid:22508339.

Deli, E., Bakle, I., \& Zachopoulou, E. (2006). Implementing intervention movement programs for kindergarten children. Journal of Early Childhood Research, 4(1), 5-18. http://dx.doi.org/10.1177/1476718X06059785.

Demets, D. L. (1987). Methods for combining randomized clinical trials: strengths and limitations. Statistics in Medicine, 6(3), 341350. http://dx.doi.org/10.1002/sim.4780060325. PMid:3616287.

Derri, V., Tsapakidou, A., Zachopoulou, E., \& Kioumourtzoglou, E. (2001). Effect of a music and movement programme on development of locomotor skills by children 4 to 6 years of age. European Journal of Physical Education., 6(1), 16-25. http://dx.doi. org/10.1080/1740898010060103.

Engel, A. C., Broderick, C. R., Van Doorn, N., Hardy, L. L., \& Parmenter, B. J. (2018). Exploring the relationship between fundamental motor skill interventions and physical activity levels in children: a systematic review and meta-analysis. Sports Medicine, 48(8), 1845-1857. http:// dx.doi.org/10.1007/s40279-018-0923-3. PMid:29687278.

Fong, S. S. M., Tsang, W. W. N., Ng, G. Y. F. (2012). Taekwondo training improves sensory organization and balance control in children with developmental coordination disorder: a randomized controlled trial. Research in Developmental Disabilities, 33(1), 85-95.

Gao, Z., Zeng, N., Pope, Z. C., Wang, R., \& Yu, F. (2019). Effects of exergaming on motor skill competence, perceived competence, and physical activity in preschool children. Journal of Sport and Health Science, 8(2), 106-113. http://dx.doi.org/10.1016/j.jshs.2018.12.001. PMid:30997256.

Hacke, C., Ketelhut, S., Wendt, U., Müller, G., Schlesner, C., \& Ketelhut, K. (2017). Effectiveness of a preschool-based exercise intervention on physical activity, motor performance, body-mass-index and blood pressure: a cluster-randomized controlled trial. Medicine and Science in Sports and Exercise, 49(5), 199. http://dx.doi.org/10.1249/01. mss.0000517384.79408.91.

Hamstra-Wright, K. L., Swanik, C. B., Sitler, M. R., Swanik, K. A., Ferber, R., Ridenour, M., \& Huxel, K. C. (2006). Gender comparisons of dynamic restraint and motor skill in children. Clinical Journal of Sport Medicine, 16(1), 56-62. http://dx.doi.org/10.1097/01. jsm.0000179232.10261.65. PMid:16377977.

Higgins, J. P. T., \& Thompson, S. G. (2002). Quantifying heterogeneity in a meta-analysis. Statistics in Medicine, 21(11), 1539-1558. http:// dx.doi.org/10.1002/sim.1186. PMid:12111919.

Higgins, J. P., \& Green, S. (2008). Cochrane handbook fo systematic reviews of interventions. Hoboken: John Wiley \& Sons.

Hung, Y., Brandão, M. B., \& Gordon, A. M. (2017). Structured skill practice during intensive bimanual training leads to better trunk and arm control than unstructured practice in children with unilateral spastic cerebral palsy. Research in Developmental Disabilities, 60, 65-76. http://dx.doi.org/10.1016/j.ridd.2016.11.012. PMid:27912104.

Iivonen, S., Sääkslahti, A., \& Nissinen, K. (2011). The development of fundamental motor skills of four- to five-year-old preschool children and the effects of a preschool physical education curriculum. Early Child Development and Care, 181(3), 335-343. http://dx.doi. org/10.1080/03004430903387461.
Ji, C. (2019). Teaching for the sake of learning: the enlightenment of $3 \mathrm{P}$ teaching model to university undergraduate education reform. Jiangsu Higher Education, (12), 109-115.

Jones, R. A., Okely, A. D., Hinkley, T., Batterham, M., \& Burke, C. (2016). Promoting gross motor skills and physical activity in childcare: a translational randomized controlled trial. Journal of Science and Medicine in Sport, 19(9), 744-749. http://dx.doi.org/10.1016/j. jsams.2015.10.006. PMid:26774378.

Jones, R. A., Riethmuller, A., Hesketh, K., Trezise, J., Batterham, M., \& Okely, A. D. (2011). Promoting fundamental movement skill development and physical activity in early childhood settings: a cluster randomized controlled trial. Pediatric Exercise Science, 23(4), 600-615. http://dx.doi.org/10.1123/pes.23.4.600. PMid:22109783.

Kang, X., Li, P., Zhang, Y., Zhang, C., \& Zhou, H. (2017). Investigation on the physical condition of preschool children in Zuojiazhuang community from 2009 to 2014. China Maternal and Child Health Care, (10), 2180-2183.

Lai, S. K., Costigan, S. A., Morgan, P. J., Lubans, D. R., Stodden, D. F., Salmon, J., \& Barnett, L. M. (2014). Do school-based interventions focusing on physical activity, fitness, or fundamental movement skill competency produce a sustained impact in these outcomes in children and adolescents? a systematic review of follow-up studies. Sports Medicine, 44(1), 67-79. http://dx.doi.org/10.1007/s40279013-0099-9. PMid:24122775.

Lemos, A. G., Avigo, E. L., \& Barela, J. A. (2012). Physical education in kindergarten promotes fundamental motor skill development. Advances in Physical Education, 2(1), 17-21. http://dx.doi.org/10.4236/ ape.2012.21003.

Lloyd, M., Saunders, T. J., Bremer, E., \& Tremblay, M. S. (2014). LongTerm Importance of Fundamental Motor Skills: A 20-Year Follow-Up Study. Adapted Physical Activity Quarterly, 31(1), 67-78.

Logan, S. W., Robinson, L. E., Wilson, A. E., \& Lucas, W. A. (2012). Getting the fundamentals of movement: a meta-analysis of the effectiveness of motor skill interventions in children. Child: Care, Health and Development, 38(3), 305-315. http://dx.doi.org/10.1111/ j.1365-2214.2011.01307.x. PMid:21880055.

Maluly, H. D. B., Johnston, C., Giglio, N. D., Schreiner, L. L., Roberts, A., \& Abegaz, E. G. (2020). Low- and no- calorie sweeteners (lncs): critical evaluation of their safety and health risks. Food Science and Technology, 40(1), 1-10. http://dx.doi.org/10.1590/fst.36818.

Meng, F., He, Q., \& Feng, Q. (2018). The relationship between 3 to 6 years old children's care and overweight and obesity in China. Chinese Public Health, (08), 1070-1074.

Moher, D., Shamseer, L., Clarke, M., Ghersi, D., Liberati, A., Petticrew, M., Shekelle, P., \& Stewart, L. A. (2015). Preferred reporting items for systematic review and meta-analysis protocols (PRISMA-P) 2015 statement. Systematic Reviews, 4(1), 1. http://dx.doi.org/10.1186/20464053-4-1. PMid:25554246.

Mostafavi, R., Ziaee, V., Akbari, H., \& Haji-Hosseini, S. (2013). The effects of SPARK physical education program on fundamental motor skills in 4-6 year-old children. Iranian Journal of Pediatrics, 23(2), 216-219. PMid:23724186.

Palmer, K. K., Chinn, K. M., \& Robinson, L. E. (2019). The effect of the CHAMP intervention on fundamental motor skills and outdoor physical activity in preschoolers. Journal of Sport and Health Science, 8(2), 98-105. http://dx.doi.org/10.1016/j.jshs.2018.12.003. PMid:30997255.

Riethmuller, A. M., Jones, R., \& Okely, A. D. (2009). Efficacy of interventions to improve motor development in young children: a systematic review abstract. Pediatrics, 124(4), e782-e792. http:// dx.doi.org/10.1542/peds.2009-0333. PMid:19736263. 
Robinson, L. E., Palmer, K. K., \& Bub, K. L. (2016). Effect of the children's health activity motor program on motor skills and self-regulation in head start preschoolers: an efficacy trial. Frontiers in Public Health, 4, 173. http://dx.doi.org/10.3389/fpubh.2016.00173. PMid:27660751.

Robinson, L. E., Stodden, D. F., Barnett, L. M., Lopes, V. P., Logan, S. W., Rodrigues, L. P., \& D’Hondt, E. (2015a). Motor competence and its effect on positive developmental trajectories of health. Sports Medicine, 45(9), 1273-1284. http://dx.doi.org/10.1007/s40279-0150351-6. PMid:26201678.

Robinson, L. E., Veldman, S. L. C., Palmer, K. K., \& Okely, A. D. (2017). A ball skills intervention in preschoolers: the CHAMP randomized controlled trial. Medicine and Science in Sports and Exercise, 49(11), 2234-2239. http://dx.doi.org/10.1249/MSS.0000000000001339. PMid:29045324.

Rocha, H. A., Marinho, D. A., Jidovtseff, B., Silva, A. J., \& Costa, A. M. (2017). Influence of regular soccer or swimming practice on gross motor development in childhood. Motricidade, 12(4), 33-43. http:// dx.doi.org/10.6063/motricidade.7477.

Stodden, D. F., Goodway, J. D., Langendorfer, S. J., Roberton, M. A., Rudisill, M. E., Garcia, C., \& Garcia, L. E. (2008). A developmental perspective on the role of motor skill competence in physical activity: an emergent relationship. Quest, 60(2), 290-306. http://dx.doi.org/ 10.1080/00336297.2008.10483582.

Stuck, A. E., Rubenstein, L. Z., Wieland, D., Vandenbroucke, J. P., Irwig, L., Macaskill, P., Berry, G., Glasziou, P., Seagroatt, V., Stratton, I., Egger, M., Smith, G. D., Minder, C., Langhorne, P., Song, F., \& Gilbody, S. (1998). Bias in meta-analysis detected by a simple, graphical test. Asymmetry detected in funnel plot was probably due to true heterogeneity. BMJ, 316(7129), 469-471. http://dx.doi. org/10.1136/bmj.316.7129.469. PMid:9492685.

Tortella, P., Haga, M., Loras, H., Sigmundsson, H., \& Fumagalli, G. (2016). Motor skill development in italian pre-school children induced by structured activities in a specific playground. PLoS One, 11(7), e0160244. http://dx.doi.org/10.1371/journal.pone.0160244. PMid:27462985.

Van Capelle, A., Broderick, C. R., Van Doorn, N., Ward, R. E., \& Parmenter, B. J. (2017). Interventions to improve fundamental motor skills in pre-school aged children: A systematic review and meta-analysis. Journal of Science and Medicine in Sport, 20(7), 658666. http://dx.doi.org/10.1016/j.jsams.2016.11.008. PMid:28169146.

Veldman, S. L. C., Palmer, K. K., Okely, A. D., \& Robinson, L. E. (2017). Promoting ball skills in preschool-age girls. Journal of Science and Medicine in Sport, 20(1), 50-54. http://dx.doi.org/10.1016/j. jsams.2016.04.009. PMid:27283343.

Wang, Z., Li, H., \& Zhang, L. (2018). A systematic review of the influence of motor ability on daily physical activity levels of children and adolescents. Chinese School Health, (10), 1472-1475.

Wasenius, N. S., Grattan, K. P., Harvey, A. L. J., Naylor, P. J., Goldfield, G. S., \& Adamo, K. B. (2018). The effect of a physical activity intervention on preschoolers' fundamental motor skills - a cluster RCT. Journal of Science and Medicine in Sport, 21(7), 714-719. http:// dx.doi.org/10.1016/j.jsams.2017.11.004. PMid:29150312.

Wen, J. Interaction of sleep and physical activity on cognitive ability of preschool children. Shanghai: Shanghai Institute of Physical Education, 2017.

Wick, K., Leeger-Aschmann, C. S., Monn, N. D., Radtke, T., Ott, L. V., Rebholz, C. E., Cruz, S., Gerber, N., Schmutz, E. A., Puder, J. J., Munsch, S., Kakebeeke, T. H., Jenni, O. G., Granacher, U., \& Kriemler, S. (2017). Interventions to promote fundamental movement skills in childcare and kindergarten: a systematic review and meta-analysis. Sports Medicine, 47(10), 2045-2068. http://dx.doi.org/10.1007/ s40279-017-0723-1. PMid:28386652.

Williams, H. G., Pfeiffer, K. A., O’Neill, J. R., Dowda, M., McIver, K. L., Brown, W. H., \& Pate, R. R. (2008). Motor skill performance and physical activity in preschool children. Obesity, 16(6), 1421-1426. http://dx.doi.org/10.1038/oby.2008.214. PMid:18388895.

Wu, S. (2015). An empirical study of rhythmic physical activity on the development of children's gross motor development from the perspective of movement development. Journal of Beijing Sport University, 38(15), 98-106.

Xin, F., Cai, Y., Bao, R., Wang, K., Chen, S., Liu, Y., \& Sun, X. (2019). A review of foreign children's basic motor skills intervention research system. Sports Science., 39(02), 83-97.

Xiong, S., Li, X., \& Tao, K. (2017). Effects of structured physical activity program on chinese young children's executive functions and perceived physical competence in a day care center. Biomed Research International, 2017, 5635070.

Yang, Q. (2017). Experimental intervention on the movement development of preschool children's large muscles from the perspective of movement development. Journal of Xian Institute of Physical Education, 34(3), 341-347.

Zhu, G. (2015). Meta-analysis of the effect of regular aerobic exercise on adult plasma interleukin-6 levels. Sports Science, 35(10), 90-98. 